\title{
Noncontact and noninvasive study of plant leaves using air-coupled ultrasounds
}

\author{
T. E. Gómez Álvarez-Arenas, ${ }^{1, a)}$ D. Sancho-Knapik, ${ }^{2}$ J. J. Peguero-Pina, ${ }^{2}$ and \\ E. Gil-Pelegrín ${ }^{2}$ \\ ${ }^{1}$ Instituto de Acústica, CSIC, Serrano 144, 28006 Madrid, Spain \\ ${ }^{2}$ Unidad de Recursos Forestales, CITA, Gobierno de Aragón, Apdo. 727, 50080 Zaragoza, Spain
}

(Received 3 July 2009; accepted 21 October 2009; published online 11 November 2009)

\begin{abstract}
Plant leaves are studied by the analysis of the magnitude and phase spectra of their thickness mechanical resonances. These resonances appear at ultrasonic frequencies and have been excited and sensed using air-coupled ultrasounds. In spite of the complex leaf microstructure, the effective medium approach can be applied to solve the inverse problem, at least in the vicinity of the first thickness resonance. Results suggest that these resonances are sensitive to leaf microstructure, composition water content and water status in the leaf. () 2009 American Institute of Physics. [doi:10.1063/1.3263138]
\end{abstract}

Plant leaves are key elements of the structure of life as it is there where the photosynthesis takes places. In addition, they are the last stage of an important and intriguing plant mechanism that permits to lift water from the soil up to the canopy. This is commonly described by the cohesion-tension theory, recently been tested in synthetic trees. ${ }^{1}$ The knowledge of the way plant leaves operate, its mechanical and microstructural properties can be useful for the design of biologically inspired materials and is attracting a growing interest. $^{2-5}$

In the context of plant physiology, it is extremely interesting to develop noninvasive procedures to monitor the dynamic of water movement through the plant in the context of the plant-soil-atmosphere continuum model. ${ }^{6}$ Applications in this field demand sensors capable of monitoring water in the plant directly and online. Available techniques present serious difficulties as they are either too laborious or the equipment are too complex or automation is not possible or they are not fully noninvasive (see Ref. 7).

Ultrasonic techniques have shown very strong capabilities for materials noninvasive and nondestructive inspection. These techniques have also been applied to study plant leaves. ${ }^{8,9}$ However, these techniques cannot be used to study variations of the water content or the turgor pressure in the leaf for water immersion was used. Use of air-coupled ultrasounds is promising. Among many different applications, aircoupled ultrasounds have been used for the study of synthetic membranes. ${ }^{10,11}$ As these membranes exhibit some similarities with plant leaves, try to adapt that technique to plant leaves seems reasonable. Toward this end, it was necessary to modify the technique to avoid the necessity to independently measure the thickness, which is not acceptable in this case.

Let us consider two transducers located facing each other. First, transmission from transmitter directly into the receiver is measured, both magnitude and phase spectra $\left(I_{0}(\omega)\right.$ and $\phi_{0}(\omega)$, respectively- $\omega$ : angular frequency- $)$ are calculated and stored. Then the leaf is put in between the transducers at normal incidence. Magnitude $-I(\omega)$ - and phase $-\phi(\omega)$ - spectra of the transmitted signal are again calculated.

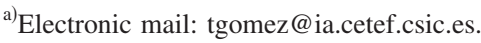

Hence, the insertion loss $\left[\mathrm{M}=I(\omega) / I_{0}(\omega)\right]$ and the phase shift $\left[\Phi=\Delta \phi=\phi(\omega)-\phi_{0}(\omega)\right]$ are obtained.

The ratio $(\xi)$ of transmitted to incident wave potentials for normal incidence on a homogeneous and isotropic plate can be analytically calculated (see, for example, Refs 10-12). Transmission coefficient $(T)$, is defined as the ratio of transmitted to incident energy fluxes, and is given by $T$ $=|\xi|^{2}$. The plot of $T$ versus frequency $-T(f)$ - shows a pattern of thickness resonances located at:

$$
\omega_{n}=2 \pi v\left(\omega_{n}\right) n / 2 h, \quad n=1,2,3, \ldots,
$$

where $v$ is the velocity in the plate and $h$ is the thickness. For our experimental set-up, following relations hold: $T=M^{2}$ and $\Phi=\phi(\xi)-h \omega / v_{a}$, where $\phi(\xi)$ is the phase of $\xi$.

If $h$ is known and $T(f)$ is measured (at least one resonance is observed), solution of the inverse problem permits us to obtain the following: $v, \alpha$, and density. ${ }^{10,11}$ On the contrary, when reverberations in the plate are either negligible or can be filtered out, then $v(\omega)$ can be obtained directly from $\Delta \phi, h$, and $v_{f}$ (the velocity of ultrasounds in the fluid where the plate is immersed $)^{13,14}$

$$
v(\omega)=h /\left(h / v_{f}-\Delta \varphi / \omega\right) .
$$

Presence of reverberations inside the plate introduces a phase shift and $\xi$ becomes a complex magnitude. ${ }^{12}$ This invalidates the use of Eq. (2). However, at $\omega=\omega_{n}$ and if dissipation in the plate can be neglected, $\xi$ is real valued, and both phase spectra with and without reverberations coincide. Therefore, Eq. (2) holds at resonance. From Eqs. (1) and (2) and measured $\omega_{1}$ and $\Delta \phi_{1}$, velocity $(v)$ and thickness $\left(h_{u s}\right)$ can be obtained. $\omega_{1}$ can be measured from the frequency location of either the maximum of $M$ or the point of inflection of $\Delta \phi$. Attenuation coefficient at $\omega_{1}=2 \pi f_{1},-\alpha\left(f_{1}\right)$-is calculated from the measured $Q$-factor of the resonance and the density is calculated from the minimum value of $T$, as explained in Refs 10 and 11. These data are used to calculate the theoretical prediction for $T$. Fitting of the theoretical values into the experimental data is performed by considering a power law for the variation of the attenuation coefficient; $\alpha(f)=\alpha\left(f_{1}\right)$ $\times\left(f / f_{1}\right)^{m}, 1<m<2$, commonly used for different viscoelastic and porous materials. ${ }^{15}$ Then, $m$ is the unique fitting pa- 


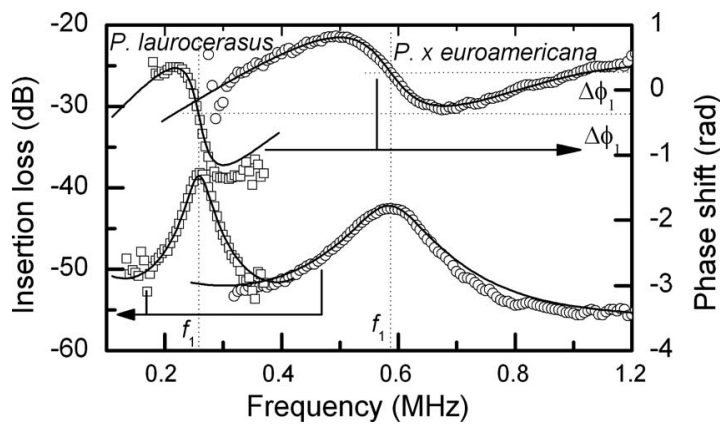

FIG. 1. Theoretical (solid line) and experimental insertion loss and phase shift vs frequency for a $P$. Laurocerasus and a $P$. $x$ Euroamericana leaf.

rameter. This nonnegligible attenuation may displace the resonant frequency away from the condition given in Eq. (1), this may require to recalculate the values of $v$ and $h$ along the fitting procedure.

Ten different leaves of two evergreen species: Prunus laurocerasus and Ligustrum lucidum and two deciduous: Platanus $x$ hispanica and Populus $x$ euroamericana were studied. The leaf life-span influences the composition of the leaf; a longer life-span implies a higher percentage of resinous substances in order to make the leaf harder and more resistant; this feature is expected to affect the leaf ultrasonic properties. Average densities were; $780 \mathrm{Kg} / \mathrm{m}^{3}$, $950 \mathrm{Kg} / \mathrm{m}^{3}, 870 \mathrm{Kg} / \mathrm{m}^{3}$, and $780 \mathrm{Kg} / \mathrm{m}^{3}$, respectively. In all cases, leaf cross-section is a four-layered structure: upper epidermis, palisade parenchyma (PP), spongy mesophyll (SM), and lower epidermis. Epidermis is a dense packing of flat cells with an external waxy cuticle, PP is a dense packaging of elongated cells (normal to the leaf plane) and the SM is a loose arrangement of cells with large intercellular spaces filled with air and moisture. Typical thickness ratio $\mathrm{SM} / \mathrm{PP}$ is approximately $\approx 2,<1, \approx 1$, and $\approx 1$ for Prunus, Ligustrum, Populus, and Platanus, respectively. The overall ultrasonic properties of the leaves are expected to be largely determined by the SM layer, where porosity is very high, $\alpha$ should be very large and $v$ very low.

Three pairs of air-coupled transducers (center frequency of $0.25,0.75$, and $1.2 \mathrm{MHz}$, circular aperture and diameter of 25,20 , and $20 \mathrm{~mm}$, respectively) were used. ${ }^{15,16}$ Transmitterreceiver separation was between 1 and $2 \mathrm{~cm}$ and leaves were located at normal incidence. Variations of the resonances when the ultrasound beam is scanned over the leaf surface were negligible. Figure 1 shows measured and calculated (assuming the leaf as a plane homogeneous and isotropic plate) $M$ and $\Delta \phi$ in the vicinity of the first thickness resonance. One interesting feature is that plant leaves, though being a complex, anisotropic, and heterogeneous material, provide a first thickness resonance that is well described by the theory for isotropic and homogeneous plates. Obtained leaf parameters from the analysis of the first thickness resonance appear in Table I. In addition, thickness was measured using a micrometer $-h_{\text {microm- }}$-(error $\left.\pm 5 \mu \mathrm{m}\right) . h_{u s}$ is very close to $h_{\text {microm}}$, differences can be accounted for by the fact that the leave deforms under the pressure of the micrometer. Table I shows, for a given specie, a certain link between leaf thickness and $v$ and $\alpha$, which suggests a close relationship between the ultrasonic parameters and either the leaf development stage or the presence of a polluted environment, which can give rise to a reduction of the PP layer. In addi-
TABLE I. Measured properties of two different leaves of each specie.

\begin{tabular}{lcccccc}
\hline \hline Sample & $\begin{array}{c}h_{\text {microm }} \\
(\mu \mathrm{m})\end{array}$ & $\begin{array}{c}h_{u s} \\
(\mu \mathrm{m})\end{array}$ & $\begin{array}{c}v(\mathrm{air}) \\
(\mathrm{m} / \mathrm{s})\end{array}$ & $\begin{array}{c}v \text { (water) } \\
(\mathrm{m} / \mathrm{s})\end{array}$ & $\begin{array}{c}f_{1} \\
(\mathrm{KHz})\end{array}$ & $\begin{array}{c}\alpha\left(f_{1}\right) \\
\mathrm{Np} / \mathrm{m}\end{array}$ \\
\hline Prunus & 425 & $464 \pm 10$ & $255 \pm 5$ & 419 & $275 \pm 4$ & $910 \pm 10$ \\
& 445 & $475 \pm 25$ & $270 \pm 15$ & 409 & $283 \pm 4$ & $800 \pm 70$ \\
Ligustrum & 345 & $370 \pm 5$ & $196 \pm 3$ & 298 & $265 \pm 4$ & $790 \pm 10$ \\
& 540 & $552 \pm 6$ & $287 \pm 3$ & 348 & $260 \pm 4$ & $416 \pm 10$ \\
Populus & 250 & $250 \pm 3$ & $365 \pm 5$ & 436 & $730 \pm 4$ & $1420 \pm 40$ \\
& 280 & $295 \pm 2$ & $351 \pm 5$ & 416 & $598 \pm 4$ & $1140 \pm 50$ \\
Platanus & 190 & $214 \pm 2$ & $275 \pm 5$ & 512 & $640 \pm 4$ & $3200 \pm 100$ \\
& 235 & $232 \pm 8$ & $369 \pm 9$ & 570 & $805 \pm 4$ & $2070 \pm 100$ \\
\hline \hline
\end{tabular}

tion, deciduous and evergreen species exhibit different ultrasonic properties.

As expected, measured $v$ and $\alpha$ are similar to those obtained for porous membranes. ${ }^{10,11}$ Under the action of the ultrasounds the fibers bend and the vacuoles deform as solid spheres in the Hertz problem. These deformation modes, common in porous media, give rise to low elastic moduli and hence, to low ultrasonic velocity. ${ }^{17}$

To determine whether the leaf layered structure has any influence on $T(f)$, Prunus and Ligustrum leaves were measured at higher frequencies, see Fig. 2. Theoretical predictions encounter some of the following problems: the periodicity of the resonances is altered and the value of $T(f)$ in between resonances is lower than predicted. This behavior is typical of a multilayered plate, where as frequency increases, resonances of sublayers may become apparent, as well as the coupling between them. It is also interesting that the increase of the damping with the frequency is much more pronounced for the Prunus than for the Ligustrum leaf. Both are evergreen species but the Prunus leave exhibit a thicker SM layer.

To determine velocity of shear waves, incidence angle of the ultrasonic beam on the leaf was changed, ${ }^{18}$ however, no evidence of shear waves was observed. Finally, the leaves were measured using a conventional water immersion and through transmission ultrasonic technique at 1.00 and 2.25 $\mathrm{MHz}$ (see Table I). Differences between ultrasonic velocities from different techniques can be explained considering that leaves rehydrates when they are immersed in water. To verify this, additional measurements were performed using a dry contact technique (at $0.25 \mathrm{MHz}$ ) on five Prunus leaves. Av-

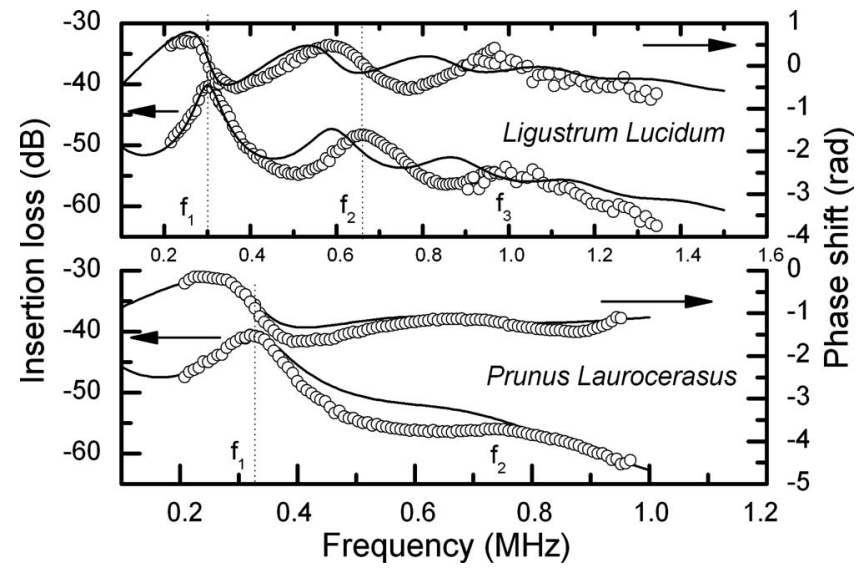

FIG. 2. Theoretical (solid line) and experimental insertion loss and phase shift vs frequency for a $P$. Laurocerasus and a L. Lucidum leaf. 


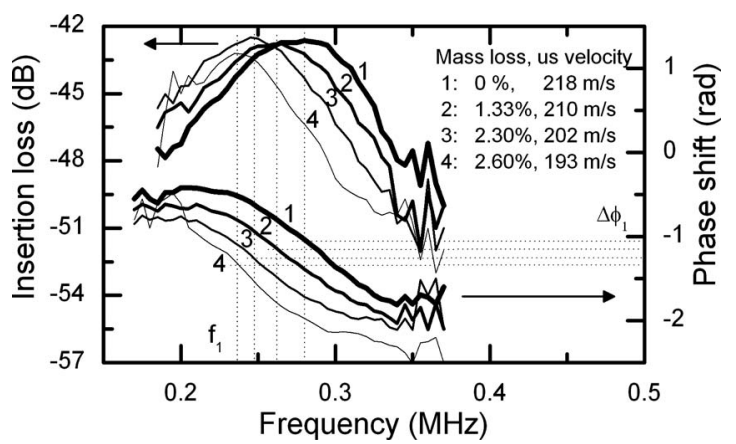

FIG. 3. Evolution of experimental insertion loss and phase shift vs frequency of a $P$. Laurocerasus leaf as water content decreases.

eraged velocity was $293 \pm 35 \mathrm{~m} / \mathrm{s}$, which agree with the value obtained with the air-coupled technique.

Finally, Fig. 3 shows that the response of the first thickness resonance of a Prunus leaf changes as it is left to dry at ambient conditions. A similar behavior was found in the other species. Loss of water is measured by weighing the leaf with a high precision balance. As water evaporates the resonance frequency and the velocity decreases while the attenuation increases. Initially (mass loss $<2.0 \%$ ) no thickness variation is observed $(400 \pm 15 \mu \mathrm{m})$, from $2.0 \%$ to $2.6 \%$ of mass loss, thickness reduces steadily down to $360 \mu \mathrm{m}$. This velocity decrease can be explained by an increase of the compressibility of the cells that build up the leaf structure due to a loss of pressure in the vacuole membrane produced by the loss of water, that is, a decrease of the turgor pressure. Calculated values of the compressibility are 37.3, 34.1, 31.25 , and $28.4 \mathrm{MPa}$, respectively.

The research presented demonstrate the possibility to excite and sense thickness resonances of plant leaves using air-coupled ultrasonic spectroscopy and to determine, simultaneously, thickness and velocity and attenuation coefficient of ultrasounds. Response of the first thickness resonance is well described by assuming the leaf as homogeneous and isotropic; deviation of higher resonance orders from this behavior can be attributed to the layered structure of the leaf.
Measurements suggest a close relationship between ultrasonic parameters and leaf development stage or environmental conditions. Evergreen and deciduous species present different ultrasonic properties that can be attributed to variations of leaf morphology and composition that depends on the leaf life-span. In addition, for similar species, the attenuation coefficient is larger and increases more rapidly with the frequency for those leaves having the thicker SM. Finally, ultrasound velocity and attenuation shows a great sensitivity to variations of the leaf water content, mainly due to the variations of turgor pressure associated to the changes in the water content.

${ }^{1}$ T. D. Wheeler and A. D. Stroock, Nature (London) 455, 208 (2008).

${ }^{2}$ R. T. Borno, J. D. Steinmeyer, and M. M. Maharbiz, Appl. Phys. Lett. 95, 013705 (2009).

${ }^{3}$ W. Barthlott and C. Neinhuis, Planta 202, 1 (1997).

${ }^{4}$ Z. M. Liu, W. J. Wu, and B. R. Hu, Sci. China, Ser. E: Technol. Sci. 51, 1902 (2008)

${ }^{5}$ Z. Guo, W. Liu, and B.-L. Su, Appl. Phys. Lett. 93, 201909 (2008).

${ }^{6} \mathrm{M}$. Tyree, Handbook of functional plant ecology (Marcel Dekker, New York, 1999), pp. 221-268.

${ }^{7}$ D. Zimmermann, R. Reuss, M. Westhoff, P. Geßner, W. Bauer, E Bamberg, F.-W. Bentrup, and U. Zimmermann, J. Exp. Bot. 59, 3157 (2008).

${ }^{8}$ M. Fukuhara, Plant Sci. 162, 521 (2002).

${ }^{9}$ P. S. Wilson and K. H. Dunton, J. Acoust. Soc. Am. 125, 1951 (2009).

${ }^{10}$ T. E. Gómez Álvarez-Arenas, J. Membr. Sci. 213, 195 (2003).

${ }^{11}$ T. E. Gómez Álvarez-Arenas, IEEE Trans. Ultrason. Ferroelectr. Freq. Control 50, 676 (2003)

${ }^{12}$ L. M. Brekhovshkihk, Waves in Layered Media (Academic Press, New York, 1960).

${ }^{13}$ T. E. Gómez Álvarez-Arenas, S. de la Fuente, and I. González, Appl. Phys. Lett. 88, 221910 (2006).

${ }^{14}$ W. Sachse and Y. H. Pao, J. Appl. Phys. 49, 4320 (1978)

${ }^{15}$ T. E. Gómez Álvarez-Arenas, IEEE Trans. Ultrason. Ferroelectr. Freq. Control 51, 624 (2004).

${ }^{16}$ T. E. Gómez Álvarez-Arenas and I. González Gómez, Appl. Phys. Lett. 90, 201903 (2007).

${ }^{17}$ L. J. Gibson and M. F. Ashby, Cellular Solids (Cambridge University Press, Cambridge, 1999), pp. 92-110.

${ }^{18}$ T. E. Gómez Álvarez-Arenas, F. Montero, M. Moner, E. Rodríguez, A Roig, and E. Molins, Appl. Phys. Lett. 81, 1198 (2002). 